\title{
Hypersensitivity pneumonitis due to isocyanate exposure in an airbag "welder"
}

\section{To the Editor:}

It is well known that exposure to isocyanate can lead to the development of asthma and is recognised as one of the major aetiologies of occupational asthma [1]. In contrast, hypersensitivity pneumonitis (HP) due to isocyanates is a rare condition.

In July 2009, a 42-yr-old female was admitted to our respiratory intensive care unit (Respiratory Dept, Centre Hospitalier Universitaire du Bocage, Dijon, France) for progressive, but severe, respiratory distress. She had no particular medical history but was a heavy smoker with a habit of 30 cigarettes a day since the age of 15 yrs. Symptoms had started 6 months earlier, with very progressive shortness of breath on exertion and dry cough. This was followed by frequent bouts of fever reaching $39^{\circ} \mathrm{C}$, chills, headaches and arthralgia without swelling. Symptoms transiently improved when she received oral corticosteroids for 7 days a month before admission. A chest radiograph performed 2 months earlier was considered normal. On admission, she was hypoxaemic, with transcutaneous saturation measured at $85 \%$, partially corrected by oxygen $\left(10 \mathrm{~L} \cdot \mathrm{min}^{-1}\right)$. Her central temperature was $39^{\circ} \mathrm{C}$. Auscultation revealed wheezing, crackles and ronchi. The laboratory examinations showed that the erythrocyte sedimentation rate $\left(68 \mathrm{~mm} \cdot \mathrm{h}^{-1}\right), \mathrm{C}$-reactive protein $\left(138 \mathrm{mg} \cdot \mathrm{L}^{-1}\right)$, fibrinogen $\left(6.4 \mathrm{~g} \cdot \mathrm{L}^{-1}\right)$ and serum lactate dehydrogenase $\left(544 \mathrm{IU} \cdot \mathrm{L}^{-1}\right)$ were all high. The white blood cell count was elevated (total: $11.5 \times 10^{3}$ cells $\cdot \mathrm{mm}^{-3}$; neutrophils:
$9.2 \times 10^{3}$ cells $\cdot \mathrm{mm}^{-3}$; lymphocytes: $1.7 \times 10^{3}$ cells $\left.\cdot \mathrm{mm}^{-3}\right)$. Arterial blood gases on room air were: arterial oxygen tension $49 \mathrm{mmHg}$; arterial carbon dioxide tension $36 \mathrm{mmHg} ; \mathrm{pH} 7.45 ; \mathrm{HCO}_{3}^{-}$ $23 \mathrm{mmol} \cdot \mathrm{L}^{-1}$. A diffuse ground-glass appearance was observed on the chest radiograph. A computed tomography scan showed bilateral patchy ground-glass opacities with air trapping in the upper fields and centrilobular ground-glass opacities predominant in the lower lobes (fig. 1). Analysis of the bronchoalveolar lavage (BAL) showed a total cell count of $640 \mathrm{cells} \cdot \mathrm{mm}^{-3}$, with $43 \%$ lymphocytes, $42 \%$ macrophages, $13 \%$ neutrophils and $2 \%$ eosinophils. She received intravenous amoxycillin-clavulanate antibiotics. As a comprehensive search for infectious disease in the BAL and blood was negative, HP was suspected. Her dyspnoea gradually improved within a few days without corticosteroids. On day 6 after admission, oxygen saturation was at $96 \%$ on $3 \mathrm{~L} \cdot \mathrm{min}^{-1}$ of oxygen at rest. Forced expiratory volume in $1 \mathrm{~s}$ (FEV1), FEV1/vital capacity and total lung capacity were $71 \%, 77 \%$ and $95 \%$ of the predicted values, respectively. Transfer coefficient of the lung for carbon monoxide (KCO) was dramatically diminished at $38 \%$ of the predicted value. We later discovered that symptom onset occurred in November 2008, 3 months after she started a job in an airbag jacket factory for horse riders or motorcyclists. She had to prepare and weld the polyurethane airbags to a security trigger device before installing the complete airbag into the jacket. Isocyanates, composing polyurethane and vaporised during airbag welding, but also cyanoacrylates contained in the
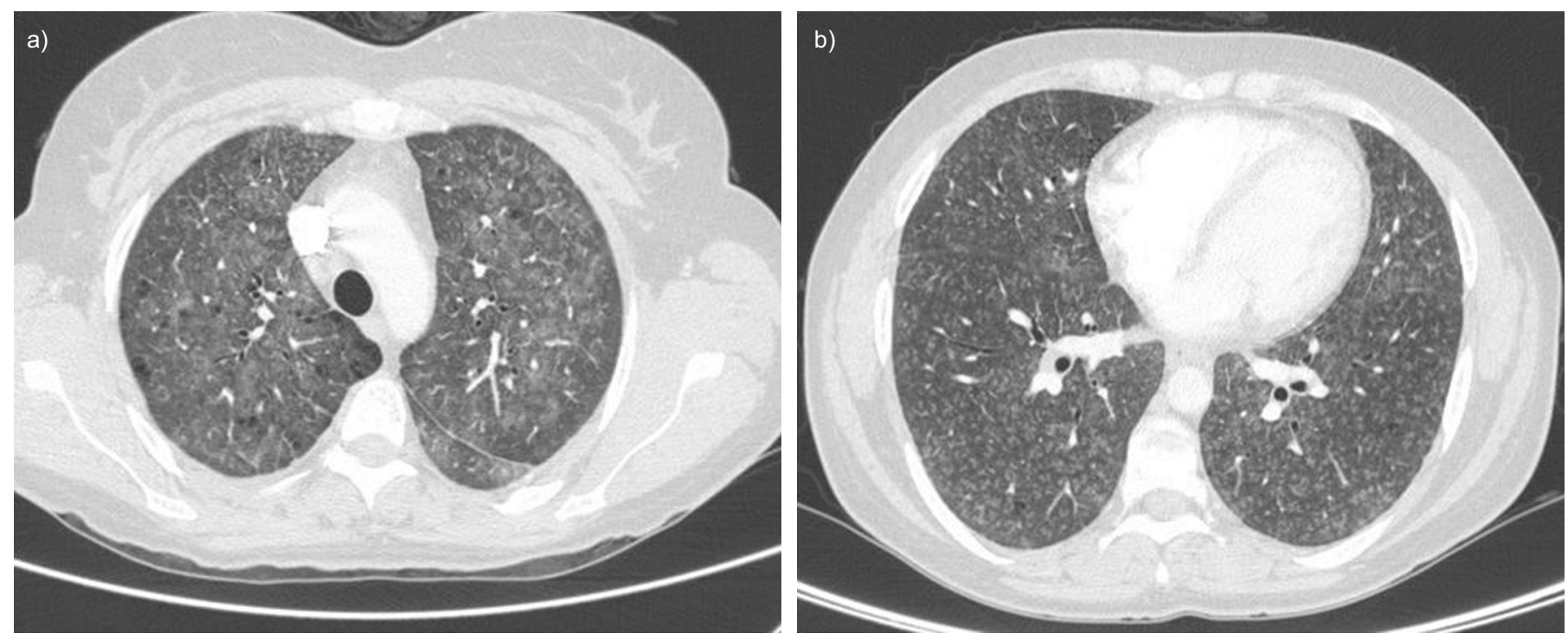

FIGURE 1. Computed tomography scan on the first day of admission. a) Upper fields: bilateral and diffuse but patchy ground-glass opacities with air trapping. b) Lower lobes: centrilobular ground-glass opacities. 
glue used to assemble the different parts of the structure, were rapidly considered as putatively responsible for this HP. Antiisocyanate specific immunoglulin $\mathrm{E}$ was negative. After her discharge from hospital, she was strongly advised not to go back to work but 2 weeks later she returned to her factory for administrative purposes. The same evening, she complained of fever and shortness of breath but did not seek medical care. A month later she was readmitted to our intensive care unit for acute respiratory distress with fever, cough and expectorations. Finger clubbing was noted. Once again, her condition improved within a few days without any specific medication. She swore she had not returned to the factory. This latest episode was later explained by the inhalation of polyurethane fumes at home due to an act of malevolence. A person, aware of her condition, was intentionally burning airbags she had stored in her garage. The absence of cyanocrylate glue during this episode definitely incriminated isocyanates. 6 months later, she no longer complained of dyspnoea. Her physical examination was normal except for the persistent clubbing. Peripheral oxygen saturation was measured at $98 \%$ on room air without dyspnoea or desaturation during a 6-min walk test $(405 \mathrm{~m})$. Chest radiographs and biology were normal. Pulmonary function tests showed no obstructive or restrictive syndrome. KCO was only $60 \%$ of the predicted value. The disease has been recognised as an occupational disease (French list $\mathrm{N}^{\circ}$ 62).

Isocyanates are low-weight molecules, characterised by the presence of at least one highly reactive isocyanate group $(\mathrm{N}=\mathrm{CO})$. Depending on the number of isocyanate groups, these molecules can be classified as mono-, di- and polyisocyanates. They interact with a hydroxyl group to form urethane and polyurethane, a major component of plastic materials. Three types of diisocyanate are commonly used in the plastics industry: toluene diisocyanate (TDI), diphenylmethane diisocyanate (MDI) and hexamethylene diisocyanate (HDI). Up to $95 \%$ of polyurethane components are produced using TDI or MDI. TDI is principally used to manufacture flexible foam for cushions and rigid insulating foam, as well as elastomers, paint coating, varnish, adhesive tape and sealant. MDI is used for the production of rigid foams, heat or sound insulation, packaging, elastomers, coating, synthetic fibres, sealants and binders for foundry stone. HDI can be found in dental prostheses, contact lenses and medical adsorbants. Isocyanates are volatile and may cause various respiratory symptoms when inhaled, such as rhinitis, bronchitis, chronic, sub-acute or acute interstitial pneumonia, and asthma, which is the most frequent manifestation. Direct toxicity ranging from local itching to extensive dermatitis may be observed on the skin and mucosa. Ocular toxicity is rare. According to some authors, sensitisation requires at least two $\mathrm{N}=\mathrm{CO}$ groups and does not occur with monoisocyanates. In contrast, a low quantity of diisocyanates or polyisocyanates may be sufficient to induce sensitisation [2]. Isocyanate-induced HP was first suspected in 1965 [3]. In guinea pigs, repeated TDI inhalation induced authentic interstitial pneumonitis on the basis of pathological findings [4]. To our knowledge, there are $<70$ suspected cases in the literature with only a dozen that fulfil all the criteria including clinical, imaging and biological findings, as well as pulmonary function test results and BAL analysis.
According to different observational occupational studies, the prevalence of isocyanate-induced HP may vary from 4 to $27 \%$ of exposed workers [5,6]. All categories of diisocyanates and polyisocyanates have been described as responsible for HP from benign forms to severe acute respiratory distress [7]. Our case matches type-II HP, as reported by LACASSE et al. [8], which is usually due to a prolonged exposure to antigens. The condition is characterised by hypoxaemia and finger clubbing associated with restrictive or obstructive symptoms and diminished KCO. The presence of anti-isocyanate specific immunoglobulin E is not necessary and not specific to HP [9].

\section{Adrien Lhoumeau*, Julien Pernot", Marjolaine Georges*, Yann Devilliers*, Jean Charles Dalphin", Philippe Camus ${ }^{*}, \boldsymbol{\sigma}_{1}+$ and Philippe Bonniaud ${ }^{*},+,+$}

* Service de Pneumologie, Centre Hospitalier Universitaire Bocage Central, "INSERM U866, University of Burgundy, ${ }^{+}$Faculty of Medicine and Pharmacy, University of Burgundy, Dijon, and "Service de Pneumologie, Centre Hospitalier Universitaire, Besançon, France.

Correspondence: P. Bonniaud, Service de Pneumologie, Centre Hospitalier Universitaire Bocage Central, Pôle Coeur Poumon Vaisseaux, 14 rue Paul Gaffarel, 21000 Dijon, France. E-mail: philippe.bonniaud@chu-dijon.fr

Statement of Interest: None declared.

Provenance: Submitted article, peer reviewed.

\section{REFERENCES}

1 Tarlo SM, Liss GM. Diisocyanate-induced asthma: diagnosis, prognosis, and effects of medical surveillance measures. Appl Occup Environ Hyg 2002; 17: 902-908.

2 Tarlo SM. Diisocyanate sensitization and antibody production. J Allergy Clin Immunol 1999; 103: 739-741.

3 Charles J, Bernstein A, Jones B, et al. Hypersensitivity pneumonitis after exposure to isocyanates. Thorax 1976; 31: 127-136.

4 Yamada K, Amitani R, Niimi A, et al. Interstitial pneumonitis-like lesions in guinea-pigs following repeated exposure to toluene diisocyanate. Eur Respir J 1995; 8: 1300-1306.

5 Baur X. Hypersensitivity pneumonitis (extrinsic allergic alveolitis) induced by isocyanates. J Allergy Clin Immunol 1995; 95: 1004-1010.

6 Simpson C, Garabrant D, Torrey S, et al. Hypersensitivity pneumonitis-like reaction and occupational asthma associated with 1,3-bis(isocyanatomethyl) cyclohexane pre-polymer. Am J Ind Med 1996; 30: 48-55.

7 Merget R, Marczynski B, Chen Z, et al. Haemorrhagic hypersensitivity pneumonitis due to naphthylene-1,5-diisocyanate. Eur Respir J 2002; 19: 377-380.

8 Lacasse Y, Selman M, Costabel U, et al. Classification of hypersensitivity pneumonitis: a hypothesis. Int Arch Allergy Appl Immunol 2009; 149: 161-166.

9 Vandenplas O, Malo JL, Saetta M, et al. Occupational asthma and extrinsic alveolitis due to isocyanates: current status and perspectives. Br J Ind Med 1993; 50: 213-228.

DOI: $10.1183 / 09059180.00008811$ 